Homology, Homotopy and Applications, vol.16(2), 2014, pp.275-288

\title{
MOTIVES AND ORIENTED COHOMOLOGY OF GENERICALLY CELLULAR VARIETIES
}

\author{
ALEXANDER NESHITOV
}

(communicated by Ulf Rehmann)

\begin{abstract}
For a cellular variety $X$ over a field $k$ of characteristic 0 and an algebraic oriented cohomology theory $\mathrm{h}$ of Levine-Morel we construct a filtration on the cohomology ring $\mathrm{h}(X)$ such that the associated graded ring is isomorphic to the Chow ring of $X$. Using this filtration we establish the following comparison result between Chow motives and h-motives of generically cellular varieties: any irreducible Chow-motivic decomposition of a generically cellular variety $Y$ gives rise to an h-motivic decomposition of $Y$ with the same generating function. Moreover, under some conditions on the coefficient ring of $\mathrm{h}$ the obtained $\mathrm{h}$ motivic decomposition will be irreducible. We also prove that if the Chow motives of two twisted forms of $Y$ coincide, then their h-motives coincide as well.
\end{abstract}

\section{Introduction}

We work over the base field $k$ of characteristic 0 . The notion of an algebraic oriented cohomology theory was studied by Levine-Morel [11] and Panin-Smirnov [15]. In this paper we will work with an oriented cohomology theory $\mathrm{h}$ in the sense of Levine-Morel introduced in $[\mathbf{1 1}, \S 1.1]$. Moreover, we assume that $\mathrm{h}$ is generically constant and has the localization property (see Definition 2.7). We denote its coefficient ring $\mathrm{h}$ (Spec $k$ ) by $\Lambda$. Let $X$ be a cellular variety with $N=\operatorname{dim} X$. We construct a filtration

$$
\mathrm{h}(X)=\mathrm{h}^{(0)}(X) \supseteq \mathrm{h}^{(1)}(X) \supseteq \cdots \supseteq \mathrm{h}^{(N)}(X) \supseteq 0
$$

on the cohomology ring such that the associated graded ring

$$
G r^{*} \mathrm{~h}(X)=\bigoplus_{i \geqslant 0} \mathrm{~h}^{(i)}(X) / \mathrm{h}^{(i+1)}(X)
$$

is isomorphic (as a graded ring) to the Chow ring $\mathrm{CH}^{*}(X, \Lambda)$ of algebraic cycles modulo rational equivalence relation with coefficients in the ring $\Lambda$. We exploit this filtration and isomorphism in the context of h-motives of generically cellular varieties. The latter is a natural generalization of the notion of Chow motives to the case of an

Received July 14, 2013, revised July7, 2014; published on October 22, 2014.

2010 Mathematics Subject Classification: 20G10, 14F43.

Key words and phrases: flag variety, oriented cohomology theory, algebraic group, algebraic cobordism.

Article available at http://dx.doi.org/10.4310/HHA.2014.v16.n2.a15

Copyright (C) 2014, International Press. Permission to copy for private use granted. 
arbitrary algebraic oriented cohomology theory of Levine-Morel. The first construction of the category of $h$-motives was introduced by Manin in [12]. This notion was also studied by Nenashev-Zainoulline in $[\mathbf{1 4}]$ and Vishik-Yagita in $[\mathbf{1 8}]$.

Let $\Lambda^{i}$ denote the $i$-th graded component of the coefficient ring $\Lambda$. We prove the following theorem that relates the $\mathrm{h}$-motive of a generically cellular variety to its Chow motive:

Theorem A. Let $X$ be a generically cellular variety over $k$, i.e., cellular over the function field $k(X)$. Assume that the Chow motive of $X$ with coefficients in $\Lambda^{0}$ splits as

$$
M^{\mathrm{CH}}\left(X, \Lambda^{0}\right)=\bigoplus_{i=1}^{n} \mathcal{R}\left(\alpha_{i}\right)
$$

for some motive $\mathcal{R}$ that splits as a direct sum of twisted Tate motives $\overline{\mathcal{R}}=\bigoplus_{j=1}^{m} \Lambda^{0}\left(\beta_{j}\right)$ over its splitting field.

Then the h-motive of $X$ splits as

$$
M^{\mathrm{h}}(X)=\bigoplus_{i=1}^{n} \mathcal{R}_{\mathrm{h}}\left(\alpha_{i}\right)
$$

for some motive $\mathcal{R}_{\mathrm{h}}$, and over the same splitting field $\mathcal{R}_{\mathrm{h}}$ splits as a direct sum of twisted h-Tate motives $\overline{\mathcal{R}_{\mathrm{h}}}=\bigoplus_{j=1}^{m} \Lambda\left(\beta_{j}\right)$.

This result can also be derived from the arguments of [18] where it is proved that sets of isomorphism classes of objects of categories of Chow motives and $\Omega$-motives coincide. However, our approach gives a more explicit correspondence between the idempotents defining the (Chow) motive $\mathcal{R}$ and the h-motive $\mathcal{R}_{\mathrm{h}}$. The latter allows us to prove the following result concerning the indecomposability of the $\mathrm{h}$-motive $\mathcal{R}_{\mathrm{h}}$ :

Theorem B. Assume that $\Lambda^{1}=\cdots \Lambda^{N}=0$, where $N=\operatorname{dim} X$.

If the Chow motive $\mathcal{R}$ is indecomposable (over $\Lambda^{0}$ ), then the h-motive $\mathcal{R}_{\mathrm{h}}$ is indecomposable (over $\Lambda$ ).

and also the following comparison property:

Theorem C. Suppose that $X, Y$ are generically cellular and $Y$ is a twisted form of $X$, i.e., $Y$ becomes isomorphic to $X$ over some splitting field.

If $M^{\mathrm{CH}}\left(X, \Lambda^{0}\right) \cong M^{\mathrm{CH}}\left(Y, \Lambda^{0}\right)$, then $M^{\mathrm{h}}(X) \cong M^{\mathrm{h}}(Y)$.

The paper is organized as follows: In section 2 we recall concepts of an algebraic oriented cohomology theory $\mathrm{h}$ of Levine-Morel and the corresponding category of $\mathrm{h}$ motives. In section 3 we introduce the filtration on the cohomology $\operatorname{ring} \mathrm{h}(X)$ of a cellular variety $X$, which plays a central role in the paper. In section 4 we apply the filtration to obtain comparison results between h-motives and Chow-motives of generically split varieties.

\section{Acknowledgments}

I am grateful to my PhD supervisor Kirill Zainoulline for useful discussions concerning the subject of this paper. The work has been supported by the Ontario Trillium Graduate Scholarship, RFBR grant 12-01-33057 and by NSERC 396100-2010 and ERA grants of K. Zainoulline. 
MOTIVES AND ORIENTED COHOMOLOGY OF GENERICALLY CELLULAR VARIETIES 277

\section{Preliminaries}

In the present section we recall the notions of algebraic oriented cohomology theory, formal group law, and cellular variety. We recall the definition of the category of $\mathrm{h}$ motives with the inverted Tate object.

\section{Oriented cohomology theories}

In this subsection we give a definition of a generically constant oriented cohomology theory with localization property (Definition 2.7). Let $\mathbf{S c h}_{k}$ denote the category of separated schemes of finite type over Spec $k=p t$, and $\mathbf{S m}_{k}$ its full subcategory of smooth quasiprojective schemes.

Definition 2.1. ([11, Definition 1.1.2])

An algebraic oriented cohomology theory $\mathbf{h}^{*}$ on $\mathbf{S m}_{k}$ is given by

(D1) Additive functor $\mathrm{h}^{*}: \mathbf{S m}_{k}^{o p} \rightarrow$ Commutative graded rings;

(D2) For every projective morphism $f: X \rightarrow Y$ of relative codimension $d$, a homomorphism of graded $\mathrm{h}^{*}(Y)$-modules $f_{\mathrm{h}}: \mathrm{h}^{*}(X) \rightarrow \mathrm{h}^{*+d}(Y)$.

These satisfy the list of axioms $(A 1),(A 2),(P B),(E H)$ of $[\mathbf{1 1}$, Definition 1.1.2].

Let us fix some notation. For a morphism $f: X \rightarrow Y$ in $\mathbf{S m}_{k}$ we will denote its image $\mathrm{h}^{*}(f): \mathrm{h}^{*}(Y) \rightarrow \mathrm{h}^{*}(X)$ by $f^{\mathrm{h}}$ and call it the pullback morphism of $f$. For a projective $f: X \rightarrow Y$ of relative codimension $d$ the morphism $f_{\mathrm{h}}: \mathrm{h}^{*}(X) \rightarrow \mathrm{h}^{*+d}(Y)$ introduced in (D2) is called the pushforward morphism of $f$.

We denote the coefficient ring $\mathrm{h}^{*}(p t)$ by $\Lambda^{*}$. As for the Chow groups, we will also use the lower grading notation for $\mathrm{h}$, i.e., $\mathrm{h}_{i}(X)=\mathrm{h}^{\operatorname{dim} X-i}(X)$ for an irreducible variety $X$.

Let $\mathbf{S c h}_{k}^{\prime}$ denote the subcategory of $\mathbf{S} c h_{k}$ consisting of projective morphisms between all the schemes in $\mathbf{S c h}_{k}$

Definition 2.2. ([11, Definition 2.1.2])

An oriented Borel-Moore functor $H_{*}$ on the category $\mathbf{S c h}_{k}$ is given by

- An additive functor $H_{*}: \mathbf{S c h}_{k}^{\prime} \rightarrow$ Graded abelian groups;

- For each smooth equidimensional morphism $f: X \rightarrow Y$ of relative dimension $d$, a homomorphism of abelian groups $f^{*}: H_{*}(Y) \rightarrow H_{*+d}(X)$;

- For each line bundle $L$ on $X$, a homomorphism of abelian groups $\widetilde{c}_{1}: H_{*}(X) \rightarrow$ $H_{*-1}(X)$.

These data satisfy the axioms (A1)-(A5) of [11, Definition 2.1.2].

An oriented Borel-Moore functor $H_{*}$ is called an oriented Borel-Moore weak homology if it additionally satisfies the axioms of [11, Definition 4.1.9].

Remark 2.3. Replacing $\mathbf{S} \mathbf{c h}$ by $\mathbf{S m}_{k}$ in the previous definition, we get the notion of an oriented Borel-Moore weak homology on $\mathbf{S m}_{k}$.

Definition 2.4. ([11, Definition 4.4.6]) The oriented Borel-Moore weak homology $H$ on $\mathbf{S c h}_{k}$ has the localization property if the following sequence is exact:

$$
H_{*}(Z) \stackrel{i_{*}}{\rightarrow} H_{*}(X) \stackrel{j^{*}}{\rightarrow} H_{*}(U) \rightarrow 0
$$

for any closed immersion $Z \rightarrow X$ and its open complement $U \rightarrow X$ in $\mathbf{S c h}_{k}$. 
Following the approach of $[\mathbf{1 1}, \S 4.4 .1]$ for a finitely generated field extension $F / k$ and for every scheme $X$ over $k$, we define the value of the oriented Borel-Moore weak homology on the scheme $X \times_{k} F$ as follows:

$$
H_{*}\left(X \times_{k} F\right)=\operatorname{colim}_{U} H_{*+\operatorname{dim} U}\left(X \times_{k} U\right),
$$

where the colimit is taken over the category whose objects are schemes $U \in \mathbf{S c h}_{k}$ such that $k(U)=F$, and whose morphisms are smooth birational maps between them. Since $k$ has characteristic zero, we may assume that all the schemes $U$ are smooth. Then we get a canonical map $H_{*}(X) \rightarrow H_{*}\left(X \times_{k} F\right)$ arising from the pullbacks $H_{*}(X) \rightarrow H_{*+\operatorname{dim} U}(X \times U)$. Note that for the case $F=k(Y)$ for $Y \in \mathbf{S m}_{k}$, the definition gives the identification $H_{*}\left(X \times_{k} k(Y)\right)=\operatorname{colim}_{U \subseteq Y} H_{*+\operatorname{dim} Y}\left(X \times_{k} U\right)$, where the colimit is taken over the category of open subsets of $Y$.

Definition 2.5. ([11, Definition 4.4.1]) The oriented Borel-Moore weak homology $H_{*}$ on $\mathbf{S c h}_{k}$ is generically constant if, for every finitely generated separable field extension $F / k$, the canonical morphism $H_{*}(k) \rightarrow H_{*}(F)$ is an isomorphism.

According to [11, Remark 5.2.7] every oriented cohomology theory $\mathrm{h}^{*}$ defines an oriented Borel-Moore weak homology on the category $\mathbf{S m}_{k}$.

Definition 2.6. We say that an oriented cohomology theory $h^{*}$ is associated to a Borel-Moore weak homology $H_{*}$ on $\mathbf{S c h}_{k}$ if the restriction of $H_{*}$ to the category $\mathbf{S m}_{k}$ coincides with the oriented Borel-Moore homology defined on $\mathbf{S m}_{k}$ by the theory $\mathrm{h}^{*}$.

Definition 2.7. An oriented cohomology theory $h^{*}$ is generically constant with a localization property if it is associated to some oriented Borel-Moore weak homology $H_{*}$ that is generically constant and satisfies the localization property.

Remark 2.8. Examples of the theories $\mathrm{h}^{*}$ defined above include Chow groups $\mathrm{CH}^{*}$, Grothendieck $K_{0}$, algebraic cobordism of Levine-Morel $\Omega^{*}$, and the theories of the form $\Omega^{*} \otimes_{\mathbb{L}} \Lambda$ given by arbitrary formal group law over the base ring $\Lambda$.

\section{Formal group law}

For an oriented cohomology theory $\mathrm{h}^{*}$ there is a notion of the first Chern class of a line bundle. For $X \in \mathbf{S m}_{k}$ and a line bundle $L$ over $X$ it is defined as $c_{1}^{\mathrm{h}}(L)=z^{\mathrm{h}} z_{\mathrm{h}}(1) \in$ $\mathrm{h}^{1}(X)$, where $z: X \rightarrow L$ is a zero section. There is a commutative associative 1dimensional formal group law $F$ over $\Lambda^{*}$ such that for any two line bundles $L_{1}$ and $L_{2}$ over $X$ we have $c_{1}^{\mathrm{h}}\left(L_{1} \otimes L_{2}\right)=F\left(c_{1}^{\mathrm{h}}\left(L_{1}\right), c_{1}^{\mathrm{h}}\left(L_{2}\right)\right)$ [11, Lem. 1.1.3]. We will use the notation $x+_{F} y$ for $F(x, y)$. For any $x$ we will denote by $-{ }_{F} x$ the unique element such that $x+{ }_{F}\left(-{ }_{F} x\right)=0$. For any $n \in \mathbb{Z}$ we will denote by $n \cdot{ }_{F} x$ the expression $x+{ }_{F} \cdots+{ }_{F} x$ ( $n$ times) if $n$ is positive, and $\left(-{ }_{F} x\right)+{ }_{F} \cdots+{ }_{F}\left(-{ }_{F} x\right)(-n$ times $)$ if $n$ is negative.

By [11] there is a universal formal group law $F_{U}$ over the Lazard ring $\mathbb{L}$. It corresponds to the algebraic cobordism theory $\Omega$ and there is a natural transformation $\Omega^{*}(-) \rightarrow \mathrm{h}^{*}(-)$ that commutes with pushforwards. This gives rise to a morphism

$$
\nu_{X}: \Omega^{*}(X) \otimes_{\mathbb{L}^{*}} \Lambda^{*} \rightarrow \mathrm{h}^{*}(X),
$$

where the ring homomorphism $\mathbb{L}^{*} \rightarrow \Lambda^{*}$ is obtained by specializing the coefficients of $F_{U}$ to the coefficients of $F$. We will call $\nu_{X}$ the specialization homomorphism. 


\section{Cellular and generically cellular varieties}

Definition 2.9. A variety $X \in \mathbf{S m}_{k}$ is called cellular if there is a filtration of $X=$ $X_{0} \supseteq X_{1} \supseteq \cdots \supseteq X_{m} \supseteq \emptyset$ such that each $X_{i} \backslash X_{i+1}$ is a disjoint union of affine spaces of the same rank $c_{i}: X_{i} \backslash X_{i+1} \cong \mathbb{A}_{k}^{c_{i}} \amalg \cdots \coprod \mathbb{A}_{k}^{c_{i}}$.

Definition 2.10. A variety $X$ is called generically cellular if $X_{k(X)}$ is a cellular variety over $k(X)$, where $k(X)$ is the function field of $X$.

Example 2.11. Let $G$ be a split semisimple algebraic group, $B$ its Borel subgroup containing a fixed maximal split torus $T$, and $W$ the corresponding Weyl group. For any $w \in W$ let $l(w)$ denote its length. Let $w_{0} \in W$ denote the longest element of $W$ and $N=l\left(w_{0}\right)$. Then the flag variety $X=G / B$ is cellular of dimension $N$ and the cellular structure is given by the Schubert cells $X_{w}$ :

$$
X=X_{w_{0}} \supseteq \bigcup_{l(w)=N-1} X_{w} \supseteq \bigcup_{l(w)=N-2} X_{w} \supseteq \cdots \supseteq X_{e}=p t,
$$

where $X_{w}$ is the closure of $B w B / B$ in $X$.

Example 2.12. Let $\zeta \in Z^{1}(k, G)$ be a 1-cocycle with values in $G$. Then the twisted form ${ }_{\zeta}(G / B)$ of $X=G / B$ provides an example of a generically split variety.

\section{h-motives}

The notion of $\mathrm{h}$-motives for the algebraic oriented cohomology theory $\mathrm{h}$ first appeared in [12]. Later it was studied in [14] and [18]. We refer to [18, §2] for the definition of the category of effective h-motives. In the present paper we will deal with the category of h-motives $\mathcal{M}_{\mathrm{h}}$ with the inverted Tate object. It is constructed as follows:

Let $\mathbf{S m P r o j}_{k}$ denote the category of smooth projective varieties over $k$. Following [5], we consider the category $\operatorname{Corr}_{\mathrm{h}}$ defined as follows: For $X, Y \in \mathbf{S m P r o j}_{k}$ with irreducible $X$ and $m \in \mathbb{Z}$ we set

$$
\operatorname{Corr}_{m}(X, Y)=\mathrm{h}_{\operatorname{dim} X+m}(X \times Y) .
$$

Objects of $\operatorname{Corr}_{\mathrm{h}}$ are pairs $(X, i)$ with $X \in \mathbf{S m P r o j}_{k}$ and $i \in \mathbb{Z}$. For $X \in \mathbf{S m P r o j}_{k}$ with irreducible components $X_{l}$ define the morphisms

$$
\operatorname{Hom}_{\text {Corr }}((X, i),(Y, j))=\coprod_{l} \operatorname{Corr}_{i-j}\left(X_{l}, Y\right) .
$$

For $\alpha \in \operatorname{Hom}((X, i),(Y, j))$ and $\beta \in \operatorname{Hom}((Y, j),(Z, k))$ the composition is given by the usual correspondence product: $\alpha \circ \beta=\left(p_{X Z}\right)_{\mathrm{h}}\left(\left(p_{Y Z}\right)^{\mathrm{h}}(\beta) \cdot\left(p_{X Y}\right)^{\mathrm{h}}(\alpha)\right)$, where $p_{X Y}, p_{Y Z}, p_{X Z}$ denote the projections from $X \times Y \times Z$ onto the corresponding summands.

Taking consecutive additive and idempotent completion of $\operatorname{Corr}_{\mathrm{h}}$ we obtain the category $\mathcal{M}_{\mathrm{h}}$ of h-motives with inverted Tate object. Objects of this category are $\left(\coprod_{i}\left(X_{i}, n_{i}\right), p\right)$ where $p$ is a matrix with entries $p_{i, j} \in \operatorname{Corr}_{n_{i}-n_{j}}\left(X_{i}, X_{j}\right)$ such that $p \circ$ $p=p$. Morphisms between $\left(\amalg\left(X_{i}, n_{i}\right), p\right)$ and $\left(\amalg\left(Y_{j}, m_{j}\right), q\right)$ are given by the set $q \circ$ $\bigoplus_{i, j} \operatorname{Corr}_{n_{i}-m_{j}}\left(X_{i}, Y_{j}\right) \circ p$ considered as a subset of $\bigoplus_{i, j} \operatorname{Corr}_{n_{i}-m_{j}}\left(X_{i}, Y_{j}\right)$. This is an additive category where each idempotent splits. There is a functor

$$
M^{\mathrm{h}}: \operatorname{SmProj}_{k} \rightarrow \mathcal{M}_{\mathrm{h}}
$$


that maps a variety $X$ to the motive $M^{\mathrm{h}}(X)=\left((X, 0), i d_{X}\right)$ and any morphism $f: X \rightarrow Y$ to the correspondence $\left(\Gamma_{f}\right)_{\mathrm{h}}(1) \in \mathrm{h}_{\operatorname{dim} X}(X \times Y)=\operatorname{Corr}_{0}(X, Y)$, where $\Gamma_{f}: X \rightarrow X \times Y$ is the graph inclusion. We will denote by $\Delta: X \rightarrow X \times X$ the diagonal embedding. Then $\Delta_{\mathrm{h}}(1)$ is the identity in $\operatorname{Corr}_{0}(X, X)$.

We will denote Spec $k$ by $p t$ and its motive $M^{\mathrm{h}}(p t)$ by $\Lambda$. The $\mathrm{h}$-Tate motive $\Lambda(1)$ is defined as $\left((p t, 1), i d_{p t}\right)$. We write $\Lambda(n)$ for $\Lambda(1)^{\otimes n}$ and $M^{\mathrm{h}}(X)(n)$ for $M^{\mathrm{h}}(X) \otimes \Lambda(n)$. The motive $M^{\mathrm{h}}(X)(n)$ is called the $n$-th twist of the motive $M^{\mathrm{h}}(X)$.

By definition we have

$$
\mathrm{h}^{i}(X)=\operatorname{Hom}_{\mathcal{M}_{\mathrm{h}}}\left(M^{\mathrm{h}}(X), \Lambda(i)\right) \text { and } \mathrm{h}_{i}(X)=\operatorname{Hom}_{\mathcal{M}_{\mathrm{h}}}\left(\Lambda(i), M^{\mathrm{h}}(X)\right) .
$$

Lemma 2.13. For $X \in \mathbf{S m P r o j}_{k}$ with the structure morphism $\pi: X \rightarrow p t$, a choice of an isomorphism $M^{\mathrm{h}}(X) \cong \bigoplus_{i=1}^{n} \Lambda\left(\alpha_{i}\right)$ is equivalent to a choice of two $\Lambda$-basis sets

$$
\left\{\tau_{i} \in \mathrm{h}^{\alpha_{i}}(X)\right\}_{i} \text { and }\left\{\zeta_{i} \in \mathrm{h}_{\alpha_{i}}(X)\right\}_{i}
$$

such that $\pi_{\mathrm{h}}\left(\tau_{i} \zeta_{j}\right)=\delta_{i, j}$ in $\Lambda$ and $\sum_{i} \zeta_{i} \otimes \tau_{i}=\Delta_{\mathrm{h}}(1)$ in $\mathrm{h}(X \times X)$.

Proof. In the decomposition $M^{\mathrm{h}}(X) \cong \bigoplus_{i} \Lambda\left(\alpha_{i}\right)$ the $i$-th projection $p_{i}: M^{\mathrm{h}}(X) \rightarrow$ $\Lambda\left(\alpha_{i}\right)$ is defined by an element $\tau_{i} \in \mathrm{h}^{\alpha_{i}}(X)$ and the $i$-th inclusion $\imath_{i}: \Lambda\left(\alpha_{i}\right) \rightarrow \mathrm{h}(X)$ is defined by an element $\zeta_{i} \in \mathrm{h}_{\alpha_{i}}(X)$. Then the identities $p_{i} \circ \imath_{j}=\delta_{i, j}$ and $\sum \imath_{i} \circ p_{i}=$ $i d_{M^{\mathrm{h}}(X)}$ are equivalent to the equalities $\pi_{\mathrm{h}}\left(\tau_{i} \zeta_{j}\right)=\delta_{i, j}$ and $\sum_{i=1}^{n} \zeta_{i} \otimes \tau_{i}=\Delta_{\mathrm{h}}(1)$. Let us check that $\left\{\zeta_{i}\right\}_{i}$ form a basis of $\mathrm{h}(X)$. Indeed, we have

$$
\begin{aligned}
\mathrm{h}^{*}(X) & =\bigoplus_{j \in \mathbb{Z}} \mathrm{h}^{j}(X)=\bigoplus_{j \in \mathbb{Z}} \operatorname{Hom}_{\mathcal{M}_{\mathrm{h}}}\left(M^{\mathrm{h}}(X), \Lambda(j)\right) \\
& \cong \bigoplus_{j \in \mathbb{Z}} \operatorname{Hom}_{\mathcal{M}_{\mathrm{h}}}\left(\bigoplus_{i=1}^{n} \Lambda\left(\alpha_{i}\right), \Lambda(j)\right)=\oplus_{j \in \mathbb{Z}} \bigoplus_{i=1}^{n} \Lambda_{\alpha_{i}-j}=\bigoplus_{i=1}^{n} \Lambda_{\alpha_{i}-*}=\bigoplus_{i=1}^{n} \Lambda^{*-\alpha_{i}}
\end{aligned}
$$

and $\zeta_{i}$ are the images of standard generators. So $\left\{\zeta_{i}\right\}_{i=1}^{n}$ form a $\Lambda$-basis of $\mathrm{h}(X)$. Finally, since $\tau_{i}$ are dual to $\zeta_{i},\left\{\tau_{i}\right\}_{i=1}^{n}$ is also a basis.

\section{Filtration on the cohomology ring}

In the present section we construct a filtration on the oriented cohomology $\mathrm{h}(X)$ of a cellular variety $X$ with $\operatorname{dim} X=N$, which will play an important role in the sequel.

Proposition 3.1. Assume that $X$ is a cellular variety over $k$. Then

(1) the $\mathrm{h}$-motive of $X$ splits as $M^{\mathrm{h}}(X)=\bigoplus_{i=1}^{n} \Lambda\left(\alpha_{i}\right)$;

(2) the Künneth formula holds, i.e., the natural map $\mathrm{h}(X) \otimes_{\Lambda} \mathrm{h}(X) \rightarrow \mathrm{h}(X \times X)$ is an isomorphism;

(3) the specialization maps $\nu_{X}: \Omega(X) \otimes \Lambda \rightarrow \mathrm{h}(X)$ and $\nu_{X \times X}: \Omega(X \times X) \otimes \Lambda \rightarrow$ $\mathrm{h}(X \times X)$ are isomorphisms.

Proof. By [5, Cor. 66.4] the Chow motive $M^{\mathrm{CH}}(X)$ splits: $M^{\mathrm{CH}}(X)=\oplus_{i=1}^{n} \mathbb{Z}\left(\alpha_{i}\right)$. Then [18, Cor. 2.9] implies that the motive $M^{\Omega}(X)$ splits into a sum of twisted Tate motives $M^{\Omega}(X)=\bigoplus_{i=1}^{n} \mathbb{L}\left(\alpha_{i}\right)$. By Lemma 2.13 there are elements $\zeta_{i}^{\Omega} \in \Omega_{\alpha_{i}}(X)$ and $\tau_{i}^{\Omega} \in \Omega^{\alpha_{i}}(X)$ such that $\pi_{\Omega}\left(\zeta_{i}^{\Omega} \tau_{j}^{\Omega}\right)=\delta_{i, j}$ and $\Delta_{\Omega}(1)=\sum_{i} \zeta_{i}^{\Omega} \otimes \tau_{i}^{\Omega}$. Denote $\zeta_{i}^{\mathrm{h}}=$ $\nu_{X}\left(\zeta_{i}^{\Omega} \otimes 1\right)$ and $\tau_{i}^{\mathrm{h}}=\nu_{X}\left(\tau_{i}^{\Omega} \otimes 1\right)$. Since $\nu_{X}$ commutes with pullbacks and pushforwards, $\pi_{\mathrm{h}}\left(\zeta_{i}^{\mathrm{h}} \tau_{j}^{\mathrm{h}}\right)=\delta_{i, j}$ and $\Delta_{\mathrm{h}}(1)=\sum_{i} \zeta_{i}^{\mathrm{h}} \otimes \tau_{i}^{\mathrm{h}}$. Then by Lemma 2.13 we have $M^{\mathrm{h}}(X)=\bigoplus_{i} \Lambda\left(\alpha_{i}\right)$, so (1) holds. 
MOTIVES AND ORIENTED COHOMOLOGY OF GENERICALLY CELLULAR VARIETIES 281

The Künneth map fits into the diagram

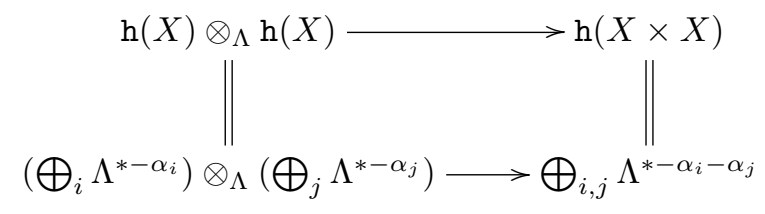

where the bottom arrow is an isomorphism, so the Künneth formula (2) holds.

Note that the natural map $\nu_{X}$ can be factored as follows

$$
\Omega(X) \otimes \Lambda \cong \oplus_{m} H_{o m} m_{\mathcal{M}_{\Omega \otimes \Lambda}}\left(\bigoplus \Lambda\left(\alpha_{i}\right), \Lambda(m)\right) \stackrel{\cong}{\rightrightarrows} \operatorname{Hom}_{\mathcal{M}_{\mathrm{h}}}\left(\bigoplus \Lambda\left(\alpha_{i}\right), \Lambda(m)\right) \cong \mathrm{h}(X) .
$$

Thus $\nu_{X}$ is an isomorphism. The same reasoning proves the statement for $\nu_{X \times X}$; hence, (3) holds.

Definition 3.2. Let $X$ be a cellular variety. Fix two basis sets $\zeta_{i}^{\Omega} \in \Omega_{\alpha_{i}}(X)$ and $\tau_{i}^{\Omega} \in \Omega^{\alpha_{i}}(X)$ provided by Proposition 3.1 and Lemma 2.13. Then $\zeta_{i}=\nu\left(\zeta_{i}^{\Omega} \otimes 1\right)$ and $\tau_{i}=\nu\left(\tau_{i}^{\Omega} \otimes 1\right)$ is a dual basis for $\mathrm{h}(X)$ as in 2.13. We define the filtration $\mathrm{h}^{(l)}(X)$ as the $\Lambda$-linear span

$$
\mathrm{h}^{(l)}(X)=\bigoplus_{N-\alpha_{i} \geqslant l} \Lambda \zeta_{i}=\bigoplus_{\alpha_{i} \geqslant l} \Lambda \tau_{i}
$$

We denote $\mathrm{h}_{N-l}^{(l)}(X)=\mathrm{h}^{(l)}(X) \cap \mathrm{h}_{N-l}(X)$ and $\mathrm{h}^{(l / l+1)}(X)=\mathrm{h}^{(l)}(X) / \mathrm{h}^{(l+1)}(X)$ and $\mathrm{h}_{N-l}^{(l / l+1)}(X)$ to be the image of $\mathrm{h}_{N-l}^{(l)}(X)$ in $\mathrm{h}^{(l / l+1)}(X)$. Lemma 3.4 implies that the latter is a graded ring.

Remark 3.3. In the case when the theory $\mathrm{h}$ is generically constant and satisfies the localization property, the filtration introduced above coincides with the topological filtration on $\mathrm{h}(X)$, i.e., with the filtration where the $l$-th term is generated over $\Lambda$ by classes $[Z \rightarrow X]$ of projective morphisms $Z \rightarrow X$ birational on its image and $\operatorname{dim} X-$ $\operatorname{dim} Z \leqslant l$. This fact follows from the generalized degree formula [11, Thm. 4.4.7].

Lemma 3.4. $\mathrm{h}^{\left(l_{1}\right)}(X) \cdot \mathrm{h}^{\left(l_{2}\right)}(X) \subseteq \mathrm{h}^{\left(l_{1}+l_{2}\right)}(X)$.

Proof. We have $\tau_{i}^{\Omega} \tau_{j}^{\Omega}=\sum_{l} a_{l} \zeta_{l}^{\Omega}$ in $\Omega(X)$ for some $a_{l} \in \mathbb{L}$. Then $\alpha_{i}+\alpha_{j}=\operatorname{deg}\left(a_{l}\right)+\alpha_{l}$. Since $\operatorname{deg}\left(a_{l}\right) \leqslant 0, \alpha_{l} \geqslant \alpha_{i}+\alpha_{j} \geqslant l_{1}+l_{2}$ for any nontrivial $a_{l}$. Since $\zeta_{i}=\nu\left(\zeta_{i}^{\Omega} \otimes 1\right)$ we have $\zeta_{i} \zeta_{j}=\sum_{l}\left(a_{l} \otimes 1\right) \zeta_{l}$ with $\alpha_{l} \geqslant \alpha_{i}+\alpha_{j} \geqslant l_{1}+l_{2}$. So $\zeta_{i} \zeta_{j} \in \mathrm{h}^{\left(l_{1}+l_{2}\right)}(X)$.

Proposition 3.5. For a cellular $X$ there is a graded ring isomorphism

$$
\Psi: \bigoplus_{i=0}^{N} \mathrm{~h}^{(i / i+1)}(X) \rightarrow \mathrm{CH}^{*}(X, \Lambda) .
$$

Proof. By Proposition 3.1 it is sufficient to prove the statement for $\mathrm{h}=\Omega$. Observe that $\Omega^{(l / l+1)}(X)$ is a free $\mathbb{L}$-module with the basis $\tau_{i}^{\Omega}+\Omega^{(l+1)}(X)$ with $\alpha_{i}=l$ and $\mathrm{CH}^{i}(X, \mathbb{L})$ is a free $\mathbb{L}$-module with basis $\tau_{i}^{\mathrm{CH}}$ with $\alpha_{i}=l$. Thus the $\mathbb{L}$-module homomorphism $\Psi_{l}$ defined by

$$
\Psi_{l}\left(\tau_{i}^{\Omega}+\Omega^{(i+1)}(X)\right)=\tau_{i}^{\mathrm{CH}}
$$

is an isomorphism.

Let us check that $\Psi=\bigoplus \Psi_{l}$ preserves multiplication. For any $i, j$ we have 


$$
\tau_{i}^{\Omega} \tau_{j}^{\Omega}=\sum_{m} a_{m} \tau_{m}^{\Omega}
$$

for some $a_{m} \in \mathbb{L}$. Then for any $m$ we have $\operatorname{deg}\left(a_{m}\right)+\alpha_{m}=\alpha_{i}+\alpha_{j}$. Then in the quotient $\Omega^{\left(\alpha_{i}+\alpha_{j} / \alpha_{i}+\alpha_{j}+1\right)}(X)$ we have

$$
\tau_{i}^{\Omega} \tau_{j}^{\Omega}=\sum_{\alpha_{m}=\alpha_{i}+\alpha_{j}} a_{m} \tau_{m}^{\Omega} \bmod \Omega^{\left(\alpha_{i}+\alpha_{j}+1\right)}(X)
$$

Observe that $\mathbb{L}^{0}=\mathbb{Z}$ and for all $a_{m} \in \mathbb{L}$ such that $\operatorname{deg}\left(a_{m}\right)<0$ we have $a_{m} \otimes 1_{\mathbb{Z}}=0$ in $\mathbb{Z}$. Thus tensoring $(*)$ with $1_{\mathbb{Z}}$ we get

$$
\tau_{i}^{\mathrm{CH}} \tau_{j}^{\mathrm{CH}}=\sum_{\alpha_{m}=\alpha_{i}+\alpha_{j}}\left(a_{m} \otimes 1\right) \tau_{m}^{\mathrm{CH}}
$$

So $\Psi_{\alpha_{i}+\alpha_{j}}\left(\tau_{i}^{\Omega}+\Omega^{\left(\alpha_{i}+1\right)}(X) \cdot \tau_{j}^{\Omega}+\Omega^{\left(\alpha_{j}+1\right)}(X)\right)=\tau_{i}^{\mathrm{CH}} \cdot \tau_{j}^{\mathrm{CH}}$. Hence $\Psi$ is a graded ring isomorphism.

Lemma 3.6. $\Psi\left(\zeta_{i}+\mathrm{h}^{\left(\alpha_{i}+1\right)}(X)\right)=\zeta_{i}^{\mathrm{CH}}$.

Proof. It is sufficient to show the statement for $\mathrm{h}=\Omega^{*}$. Consider the expansion $\zeta_{i}^{\Omega}=$ $\sum a_{j} \tau_{j}^{\Omega}$ for some $a_{j} \in \mathbb{L}$ with $\operatorname{deg} a_{j}+\alpha_{j}=N-\alpha_{i}$. Since $\operatorname{deg} a_{j} \leqslant 0$ we have

$$
\zeta_{i}^{\Omega}=\sum_{\operatorname{deg} a_{j}=0} a_{j} \tau_{j}^{\Omega} \bmod \Omega^{\left(N-\alpha_{i}+1\right)}(X) .
$$

Therefore $\Psi\left(\zeta_{i}^{\Omega}+\Omega^{\left(N-\alpha_{i}+1\right)}(X)\right)=\zeta_{i}^{\mathrm{CH}}$.

Corollary 3.7. The restriction gives rise to an isomorphism $\oplus_{i=1}^{N} \Psi^{i}: \mathrm{h}_{N-i}^{(i / i+1)}(X) \rightarrow$ $\mathrm{CH}^{*}\left(X, \Lambda^{0}\right)$.

Proof. This follows from the previous lemma and the fact that $\mathrm{CH}^{*}\left(X, \Lambda^{0}\right)=\oplus \Lambda^{0} \zeta_{i}^{\mathrm{CH}}$.

\section{Applications to h-motivic decompositions}

Throughout this section we consider a generically cellular variety $X$ of dimension $N$ and an oriented cohomology theory $\mathrm{h}^{*}$ that is generically constant and is associated with weak Borel-Moore homology $\mathrm{h}_{*}$ that satisfies the localization property. These assumptions imply that the generalized degree formula of Levine-Morel hold [11, Theorem 4.4.7]. The aim of this section is to prove theorems A, B, and C of the introduction, which provide a comparison between the Chow motive $M(X)$ and the h-motive $M^{\mathrm{h}}(X)$ of $X$.

Let $L$ be a splitting field of $X$ and $\bar{X}=X \times_{\text {Spec } k}$ Spec $L$. Let $p$ denote the projection $p: \bar{X} \times \bar{X} \rightarrow X \times X$. For any $k$-scheme $Z$ we denote by $Z_{L}$ the product $Z_{L}=Z \times \times_{\text {Spec } k}$ Spec $L$. Since $\bar{X}$ is cellular, we may consider the filtration on $\mathrm{h}(\bar{X})$ introduced in Definition 3.2. It gives rise to a filtration on $\mathrm{h}(\bar{X} \times \bar{X})=\mathrm{h}(\bar{X}) \otimes_{\Lambda} \mathrm{h}(\bar{X})$. Namely, we set

$$
\mathrm{h}^{(l)}(\bar{X} \times \bar{X})=\sum_{i+j=l} \mathrm{~h}^{(i)}(\bar{X}) \otimes_{\Lambda} \mathrm{h}^{(j)}(\bar{X}) .
$$


MOTIVES AND ORIENTED COHOMOLOGY OF GENERICALLY CELLULAR VARIETIES 283

On $\mathrm{h}(X \times X)$ we consider the induced filtration

$$
\mathrm{h}^{(l)}(X \times X)=\left(p^{\mathrm{h}}\right)^{-1}\left(\mathrm{~h}^{(l)}(\bar{X} \times \bar{X})\right) .
$$

Denote the quotient $\mathrm{h}^{(l)}(\bar{X} \times \bar{X}) / \mathrm{h}^{(l+1)}(\bar{X} \times \bar{X})$ by $\mathrm{h}^{(l / l+1)}(\bar{X} \times \bar{X})$ and denote by $p r_{l}: \mathrm{h}^{(l)}(\bar{X} \times \bar{X}) \rightarrow \mathrm{h}^{(l / l+1)}(\bar{X} \times \bar{X})$, the usual projection. Denote

$$
\begin{gathered}
\mathrm{h}_{2 N-i}^{(i)}(X \times X)=\mathrm{h}^{(i)}(X \times X) \cap \mathrm{h}_{2 N-i}(X \times X) \text { and } \\
\mathrm{h}_{2 N-i}^{(i)}(\bar{X} \times \bar{X})=\mathrm{h}^{(i)}(\bar{X} \times \bar{X}) \cap \mathrm{h}_{2 N-i}(\bar{X} \times \bar{X}) .
\end{gathered}
$$

Lemma 4.1. There is a graded ring isomorphism

$$
\Phi: \bigoplus_{i=0}^{2 N} \mathrm{~h}^{(i / i+1)}(\bar{X} \times \bar{X}) \rightarrow \mathrm{CH}^{*}(\bar{X} \times \bar{X}, \Lambda) .
$$

Proof. By Künneth formula 3.1 we may take $\Phi=\Psi \otimes \Psi$, where $\Psi$ is defined in Proposition 3.5. Then the lemma follows from Proposition 3.5.

Analogous to Corollary 3.7 we get

Remark 4.2. The restriction of $\Phi^{i}$ gives an isomorphism $\Phi^{i}: \mathrm{h}_{2 N-i}^{(i / i+1)}(\bar{X} \times \bar{X}) \rightarrow$ $\mathrm{CH}^{i}\left(\bar{X} \times \bar{X}, \Lambda^{0}\right)$.

The following lemma provides an h-version of the Rost Nilpotence Theorem:

Lemma 4.3. The kernel of the pullback map $p^{\mathrm{h}}: \operatorname{End}\left(M^{\mathrm{h}}(X)\right) \rightarrow \operatorname{End}\left(M^{\mathrm{h}}(\bar{X})\right)$ consists of nilpotents.

Proof. Let $\mu_{-}: \Omega(-) \rightarrow \mathrm{CH}(-)$ denote the natural transformation arising from the universality of $\Omega$. Then we have that $\mu_{\bar{X} \times \bar{X}} \circ p^{\Omega}=p^{\mathrm{CH}} \circ \mu_{X \times X}$. The kernel of $p^{\mathrm{CH}}$ consists of nilpotents by [19, Prop 3.1]. By [18, Prop. 2.7] the maps $\mu_{X \times X}$ and $\mu_{\bar{X} \times \bar{X}}$ are surjective and their kernels consist of nilpotents (according to composition product). Then $\operatorname{ker} p^{\Omega}$ also consists of nilpotents. Note that $\operatorname{ker}\left(p^{\Omega}\right) \otimes \Lambda$ covers $\operatorname{ker} p^{\Omega} \otimes i d$, so the latter consists of nilpotents. Further, $p^{\mathrm{h}} \circ \nu_{X \times X}=\nu_{\bar{X} \times \bar{X}} \circ\left(p^{\Omega} \otimes i d\right)$. Since $\nu_{X \times X}$ is surjective (by the generalized degree formula) and $\nu_{\bar{X}} \times \bar{X}$ is an isomorphism (by Proposition 3.1), we have that $\operatorname{ker} p^{\mathrm{h}}$ is covered by $\operatorname{ker}\left(p^{\Omega} \otimes i d\right)$ and thus consists of nilpotents.

Lemma 4.4. We have $\mathrm{h}^{(N+i)}(\bar{X} \times \bar{X}) \circ \mathrm{h}^{(N+j)}(\bar{X} \times \bar{X}) \subseteq \mathrm{h}^{(N+i+j)}(\bar{X} \times \bar{X})$.

Proof. Consider a generator $\zeta_{m} \otimes \tau_{n} \in \mathrm{h}^{(N+i)}(\bar{X} \times \bar{X})$, where $N-\alpha_{m}+\alpha_{n} \geqslant N+i$ and $\zeta_{m^{\prime}} \otimes \tau_{n^{\prime}} \in \mathrm{h}^{(N+j)}(\bar{X} \times \bar{X})$ where $N-\alpha_{m^{\prime}}+\alpha_{n^{\prime}} \geqslant N+j$. The composition $\left(\zeta_{m} \otimes\right.$ $\left.\tau_{n}\right) \circ\left(\zeta_{m^{\prime}} \otimes \tau_{n^{\prime}}\right)=\pi_{\mathrm{h}}\left(\tau_{n} \zeta_{m^{\prime}}\right)\left(\zeta_{m} \otimes \tau_{n^{\prime}}\right)$ is nonzero iff $n=m^{\prime}$. In this case $N-\alpha_{m}+$ $\alpha_{n^{\prime}}=\left(N-\alpha_{m}+\alpha_{n}\right)+\left(N-\alpha_{m^{\prime}}+\alpha_{n^{\prime}}\right)-N \geqslant N+i+j$. Thus $\zeta_{m} \otimes \tau_{n^{\prime}}$ lies in the filtration term $\mathrm{h}^{(N+i+j)}(\bar{X} \times \bar{X})$.

Lemma 4.5. The isomorphism $\Phi^{N}: \mathrm{h}_{N}^{(N / N+1)}(\bar{X} \times \bar{X}) \rightarrow \mathrm{CH}^{N}\left(\bar{X} \times \bar{X}, \Lambda^{0}\right)$ is a ring homomorphism with respect to the composition product.

Proof. This immediately follows from the fact that $\Phi$ maps residue classes of $\zeta_{i}^{\mathrm{h}} \otimes \tau_{j}^{\mathrm{h}}$ to $\zeta_{i}^{\mathrm{CH}} \otimes \tau_{j}^{\mathrm{CH}}$. 
Lemma 4.6. Let $Y$ be a twisted form of $X$, i.e., $Y_{L} \cong X_{L}=\bar{X}$. Let $p: \bar{X} \times \bar{X} \rightarrow$ $X \times Y$ denote the projection. Then for every $m$ in the diagram

$$
\mathrm{CH}^{m}\left(X \times Y, \Lambda^{0}\right) \stackrel{p^{\mathrm{CH}}}{\rightarrow} \mathrm{CH}^{m}\left(\bar{X} \times \bar{X}, \Lambda^{0}\right) \stackrel{\Phi^{m}}{\leftarrow} \mathrm{h}_{2 N-m}^{(m / m+1)}(\bar{X} \times \bar{X}) \stackrel{p r_{m}}{\leftarrow} \mathrm{h}_{2 N-m}^{(m)}(X \times Y)
$$

we have $\operatorname{im} p^{\mathrm{CH}} \subseteq \operatorname{im} \Phi^{m} \circ p r_{m} \circ p^{\mathrm{h}}$.

Proof. Note that $\mathrm{CH}^{m}\left(X \times Y, \Lambda^{0}\right)$ is generated over $\Lambda^{0}$ by classes $i_{\mathrm{CH}}(1)$ where $i: Z \rightarrow X \times Y$, where $Z \in \mathbf{S m}_{k}$, the morphism $i$ is projective, and $i(Z)$ is a closed integral subscheme of codimension $m$ and $i: Z \rightarrow i(Z)$ is birational. Consider the Cartesian diagram

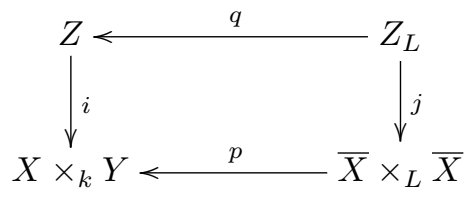

Since this diagram is transversal, we have

$$
j_{\mathrm{h}} \circ q^{\mathrm{h}}=p^{\mathrm{h}} \circ i_{\mathrm{h}} \quad \text { and } \quad j_{\mathrm{CH}} \circ q^{\mathrm{CH}}=p^{\mathrm{CH}} \circ i_{\mathrm{CH}} .
$$

By lemma 4.7 we have $j_{\mathrm{CH}}(1)=\Phi^{m} \circ p r_{m}\left(j_{\mathrm{h}}(1)\right)$. Then $p^{\mathrm{CH}}\left(i_{\mathrm{CH}}(1)\right)=\Phi^{m} \circ p r_{m} \circ$ $p^{\mathrm{h}}\left(i_{\mathrm{h}}(1)\right) \in \operatorname{im} \Phi^{m} \circ p r_{m} \circ p^{\mathrm{h}}$.

Lemma 4.7. Consider a morphism $j: Z \rightarrow \bar{X} \times_{L} \bar{X}$, where $Z \in \mathbf{S m}_{k}$, the morphism $j$ is projective, and $j(Z)$ is a closed integral subscheme of codimension $m$ and $j: Z \rightarrow$ $j(Z)$ is birational. Then $j_{\mathrm{h}}(1) \in \mathrm{h}_{2 N-m}^{(m)}(\bar{X} \times \bar{X})$ and $j_{\mathrm{CH}}(1)=\Phi^{m} \circ p r_{m}\left(j_{\mathrm{h}}(1)\right)$.

Proof. Observe that $j_{\mathrm{h}}(1)=j_{\Omega}(1) \otimes_{\mathbb{L}} 1_{\Lambda}$ and $j_{\mathrm{CH}}(1)=j_{\Omega}(1) \otimes_{\mathbb{L}} 1_{\mathbb{Z}}$. Expanding in the basis we obtain

$$
j_{\Omega}(1)=\sum_{i_{1}, i_{2}} r_{i_{1}, i_{2}} \tau_{i_{1}}^{\Omega} \otimes \tau_{i_{2}}^{\Omega} \quad \text { for some } r_{i_{1}, i_{2}} \in \mathbb{L} .
$$

Since $j_{\Omega}(1)$ is homogeneous of degree $m$, we have $r_{i_{1}, i_{2}} \in \mathbb{L}^{m-\alpha_{i_{1}}-\alpha_{i_{2}}}$. Then for every nonzero $r_{i_{1}, i_{2}}$ we have $\alpha_{i_{1}}+\alpha_{i_{2}} \geqslant m$. Then we have

$$
j_{\Omega}(1) \equiv \sum_{\alpha_{i_{1}}+\alpha_{i_{2}}=m} r_{i_{1}, i_{2}} \tau_{i_{1}}^{\Omega} \otimes \tau_{i_{2}}^{\Omega} \bmod \Omega^{(m+1)}(\bar{X} \times \bar{X}) .
$$

If $\alpha_{i_{1}}+\alpha_{i_{2}}=m$ then $r_{i_{1}, i_{2}} \in \mathbb{L}^{0}=\mathbb{Z}$. Thus taking $j_{\mathrm{h}}(1)=j_{\Omega}(1) \otimes_{\mathbb{L}} 1_{\Lambda}$ and $j_{\mathrm{CH}}(1)=$ $j_{\Omega}(1) \otimes_{\mathbb{L}} 1_{\mathbb{Z}}$ we get $\Phi^{m} \circ p r_{m}\left(j_{\mathrm{h}}(1)\right)=j_{\mathrm{CH}}(1)$, since $\tau_{i_{1}}^{\mathrm{CH}} \otimes \tau_{i_{2}}^{\mathrm{CH}}=\Phi^{m} \circ p r_{m}\left(\tau_{i_{1}}^{\mathrm{h}} \otimes \tau_{i_{2}}^{\mathrm{h}}\right)$.

Lemma 4.8. The kernel of the composition

$$
p r_{N} \circ p^{\mathrm{h}}: \mathrm{h}_{N}^{(N)}(X \times X) \rightarrow \mathrm{h}_{N}^{(N)}(\bar{X} \times \bar{X}) \rightarrow \mathrm{h}_{N}^{(N / N+1)}(\bar{X} \times \bar{X})
$$

consists of nilpotents.

Proof. This follows from Lemma 4.3 and the fact that $\mathrm{h}^{(N+1)}(\bar{X} \times \bar{X})$ is nilpotent by Lemma 4.4 .

Lemma 4.9. Let $\mathcal{C}$ be an additive category, $A, B \in O b(\mathcal{C})$. Let $f \in H_{\mathcal{C}}(A, B)$ and $g \in \operatorname{Hom}_{\mathcal{C}}(B, A)$ such that $f \circ g-i d_{B}$ is nilpotent in the ring $\operatorname{End}_{\mathcal{C}}(B)$ and $g \circ f-$ $i d_{A}$ is nilpotent in the ring $\operatorname{End}_{\mathcal{C}}(A)$. Then $A$ is isomorphic to $B$. 
Proof. Since $f g$ is a sum of identity and a nilpotent, then $f g$ is invertible in $\operatorname{End}(B)$, so $f g$ is an isomorphism, so then $f$ has a right inverse. Symmetrically, $g f$ is invertible, so then $f$ has a left inverse. Then $f$ is an isomorphism.

We are now ready to prove theorems $\mathrm{A}, \mathrm{B}$, and $\mathrm{C}$ of the introduction.

Theorem A. Suppose $X$ is generically cellular. Assume that there is a decomposition of the Chow motive with coefficients in $\Lambda^{0}$

$$
M^{\mathrm{CH}}\left(X, \Lambda^{0}\right)=\bigoplus_{i=0}^{n} \mathcal{R}\left(\alpha_{i}\right)
$$

such that over the splitting field $L$ the motive $\mathcal{R}$ equals the sum of twisted Tate motives: $\overline{\mathcal{R}}=\bigoplus_{j=0}^{m} \Lambda^{0}\left(\beta_{j}\right)$.

Then there is a h-motive $\mathcal{R}_{\mathrm{h}}$ such that

$$
M^{\mathrm{h}}(X)=\bigoplus_{i=0}^{n} \mathcal{R}_{\mathrm{h}}\left(\alpha_{i}\right)
$$

such that over the splitting field $\mathcal{R}_{\mathrm{h}}$ splits into the h-Tate motives $\overline{\mathcal{R}_{\mathrm{h}}}=\bigoplus_{j=0}^{m} \Lambda\left(\beta_{j}\right)$.

Proof. We may assume that $\alpha_{0}=0$ in $(*)$. Then each summand $\mathcal{R}\left(\alpha_{i}\right)$ equals $\left(X, p_{i}\right)$ for some idempotent $p_{i}$, and there are mutually inverse isomorphisms $\phi_{i}$ and $\psi_{i}$ of degree $\alpha_{i}$ between $\left(X, p_{0}\right)$ and $\left(X, p_{i}\right)$. So we have

- idempotents $p_{i} \in \mathrm{CH}^{N}(X \times X), \sum p_{i}=\Delta_{\mathrm{CH}}^{X}(1), p_{i} \circ p_{j}=0$ for $i \neq j$;

- isomorphisms $\phi_{i} \in p_{0} \circ \mathrm{CH}^{N+\alpha_{i}}(X \times X) \circ p_{i}$ and $\psi_{i} \in p_{i} \circ \mathrm{CH}^{N-\alpha_{i}}(X \times X) \circ$ $p_{0}$

- such that $\phi_{i} \circ \psi_{i}=p_{0}$ and $\psi_{i} \circ \phi_{i}=p_{i}$.

Consider the homomorphisms

$\mathrm{CH}^{m}\left(X \times X, \Lambda^{0}\right) \stackrel{p^{\mathrm{CH}}}{\longrightarrow} \mathrm{CH}^{m}\left(\bar{X} \times \bar{X}, \Lambda^{0}\right) \stackrel{\Phi^{m} \circ p r_{m}}{\longleftarrow} \mathrm{h}_{2 N-m}^{(m)}(\bar{X} \times \bar{X}) \stackrel{p^{\mathrm{h}}}{\longleftarrow} \mathrm{h}_{2 N-m}^{(m)}(X \times X)$.

For $m=N$ this is a ring homomorphism by Lemma 4.5. By Lemma 4.6 the elements $p^{\mathrm{CH}}\left(p_{i}\right), p^{\mathrm{CH}}\left(\phi_{i}\right)$, and $p^{\mathrm{CH}}\left(\psi_{i}\right)$ lie in the images im $\Phi^{n} \circ p r_{N} \circ p^{\mathrm{h}}, \operatorname{im} \Phi^{N+\alpha_{i}} \circ p r_{N+\alpha_{i}} \circ$ $p^{\mathrm{h}}$, and $\operatorname{im} \Phi^{N-\alpha_{i}} \circ p r_{N-\alpha_{i}} \circ p^{\mathrm{h}}$, respectively.

By Lemma 4.8 the kernel of $p r_{N} \circ p^{\mathrm{h}}: \mathrm{h}_{N}^{(N)}(X \times X) \rightarrow \mathrm{h}_{N}^{(N / N+1)}(\bar{X} \times \bar{X})$ is nilpotent. Then by [1, Prop. 27.4] there is a set of idempotents $r_{i}$ such that $\Phi^{N} \circ p r_{N} \circ$ $p^{\mathrm{h}}\left(r_{i}\right)=p^{\mathrm{CH}}\left(p_{i}\right)$ and $r_{i}$ form a full system of orthogonal idempotents, i.e., $\sum r_{i}=$ $\Delta_{\mathrm{h}}^{X}(1), r_{i} \circ r_{j}=0$ for $i \neq j$.

Let us construct the isomorphisms between h-motives $\left(X, r_{i}\right)$ and $\left(X, r_{0}\right)\left(\alpha_{i}\right)$. Let $\phi_{i}^{\prime}$ and $\psi_{i}^{\prime}$ be some elements such that $\Phi^{N+\alpha_{i}} \circ p r_{N+\alpha_{i}} \circ p^{\mathrm{h}}\left(\phi_{i}^{\prime}\right)=p^{\mathrm{CH}}\left(\phi_{i}\right)$ and $\Phi^{N-\alpha_{i}} \circ p r_{N-\alpha_{i}} \circ p^{\mathrm{h}}\left(\psi_{i}^{\prime}\right)=p^{\mathrm{CH}}\left(\psi_{i}\right)$. Then $[\mathbf{1 7}$, Lem. 2.5] implies that there are elements $\phi_{i}^{\prime \prime} \in r_{0} \mathrm{~h}_{N}^{(N)}(X \times X) r_{i}$ and $\psi_{i}^{\prime \prime} \in r_{i} \mathrm{~h}_{N}^{(N)}(X \times X) r_{0}$ such that $\phi_{i}^{\prime \prime} \psi_{i}^{\prime \prime}=r_{0}$ and $\psi_{i}^{\prime \prime} \phi_{i}^{\prime \prime}=r_{i}$. So the h-motives $\left(X, r_{i}\right)$ and $\left(X, r_{0}\right)\left(\alpha_{i}\right)$ are isomorphic. Taking $\mathcal{R}_{\mathrm{h}}=$ $\left(X, r_{0}\right)$ we obtain a decomposition

$$
M^{\mathrm{h}}(X)=\bigoplus_{i=0}^{n}\left(X, r_{i}\right)=\bigoplus_{i=0}^{n}\left(X, r_{0}\right)\left(\alpha_{i}\right)=\bigoplus_{i=0}^{n} \mathcal{R}_{\mathrm{h}}\left(\alpha_{i}\right) .
$$

Over the splitting field the motive $\overline{\mathcal{R}_{\mathrm{h}}}$ becomes isomorphic to $\left(\bar{X}, p^{\mathrm{h}}\left(r_{0}\right)\right)$ Let us 
construct an isomorphism between $\left(\bar{X}, p^{\mathrm{h}}\left(r_{0}\right)\right)$ and $\bigoplus_{j=0}^{m} \Lambda\left(\beta_{j}\right)$. Consider an isomorphism $f$ between Chow motives $f:\left(\bar{X}, p_{0}\right) \rightarrow \bigoplus_{j=0}^{m} \Lambda^{0}\left(\beta_{j}\right)$. Then $f$ is an element $f \in \oplus_{j=0}^{m} \mathrm{CH}^{\beta_{j}}(\bar{X})$. Since the map $\Psi^{N-\beta_{j}} \circ p r_{N-\beta_{j}}$ is surjective, we can lift $f$ to $\phi \in \bigoplus_{j=0}^{m} \mathrm{~h}_{N-\beta_{j}}^{\beta_{j}}(\bar{X}) \subseteq \operatorname{Hom}\left(\left(\bar{X}, r_{0}\right), \bigoplus_{j=1}^{m} \Lambda\left(\beta_{j}\right)\right)$. Analogously, lift the inverse $f^{-1}$ to $\gamma \in \oplus_{j=1}^{m} \mathrm{~h}_{\beta_{j}}^{N-\beta_{j}}(\bar{X})$. Then $\gamma \circ \phi \in \mathrm{h}_{N}^{(N)}(\bar{X} \times \bar{X})$ is mapped to identity in $\mathrm{CH}^{N}\left(\bar{X} \times \bar{X}, \Lambda^{0}\right)$, so $\gamma \circ \phi-i d$ is nilpotent according to the composition law, since it lies in the kernel of $\Phi^{N} \circ p r_{N}$. The element $\phi \circ \gamma$ lies in $\operatorname{End}\left(\oplus_{j=0}^{m} \Lambda\left(\beta_{j}\right)\right)$ so it is represented by a matrix $\left(a_{k, l}\right)_{k, l \in\{0 \ldots m\}}$ such that $a_{k, l} \in \Lambda^{\beta_{k}-\beta_{l}}$. Since $\mathrm{h}^{(N+1)}(\bar{X})=0$, we have that the composition of multiplication and pushforward

$$
\mathrm{h}^{\left(d_{1}\right)}(\bar{X}) \otimes \mathrm{h}^{\left(d_{2}\right)}(\bar{X}) \rightarrow \mathrm{h}^{\left(d_{1}+d_{2}\right)}(\bar{X}) \stackrel{\pi_{\mathrm{h}}}{\longrightarrow} \mathrm{h}(\text { Spec } k)
$$

is zero if $d_{1}+d_{2}>N$. Therefore $a_{k, l}=0$ if $\beta_{k}-\beta_{l}>0$. Note that for $k, l$ such that $\beta_{k}=\beta_{l}$, we have $a_{k, l}=\pi_{\mathrm{h}}\left(\phi_{k} \gamma_{l}\right) \in \mathrm{h}_{0}^{(N)}(\bar{X})$. Since $\mathrm{h}^{(N+1)}(\bar{X})=0$ we have that $\Psi^{(N)}: \mathrm{h}_{0}^{(N)}(\bar{X}) \rightarrow \mathrm{CH}^{N}\left(\bar{X}, \Lambda^{0}\right)$ is an isomorphism and $\pi_{\mathrm{h}}\left(\phi_{k} \gamma_{l}\right)=\pi_{\mathrm{CH}}\left(f_{k} g_{l}\right)=\delta_{k, l}$. Thus $\left(a_{k, l}\right)$ is a triangular matrix with identity on the diagonal, so it is the sum of the identity matrix and a nilpotent matrix.

So $\gamma \circ \phi-i d$ is nilpotent and $\phi \circ \gamma-i d$ is nilpotent. Then by lemma $4.9 \overline{\mathcal{R}_{\mathrm{h}}} \cong$ $\bigoplus_{j=0}^{m} \Lambda\left(\beta_{j}\right)$.

Lemma 4.10. Assume that $\Lambda^{1}=\cdots=\Lambda^{N}=0$. Then $\mathrm{h}_{N}(\bar{X} \times \bar{X}) \subseteq \mathrm{h}^{(N)}(\bar{X} \times \bar{X})$ and for the diagram of Lemma 4.6,

$\mathrm{CH}^{N}\left(X \times X, \Lambda^{0}\right) \stackrel{p^{\mathrm{CH}}}{\longrightarrow} \mathrm{CH}^{N}\left(\bar{X} \times \bar{X}, \Lambda^{0}\right) \stackrel{\Phi^{N}}{\longleftarrow} \mathrm{h}_{N}^{(N / N+1)}(\bar{X} \times \bar{X}) \stackrel{p r_{N} \circ p^{\mathrm{h}}}{\longleftarrow} \mathrm{h}_{N}^{(N)}(X \times X)$, the equality holds: $\operatorname{im} p^{\mathrm{CH}}=\operatorname{im} \Phi^{N} \circ p r_{N} \circ p^{\mathrm{h}}$.

Proof. Note that $\mathrm{h}_{N}(\bar{X} \times \bar{X})$ is additively generated by elements of the form $x=$ $\lambda \tau_{i} \otimes \tau_{j}$, where $\operatorname{deg} \lambda+\alpha_{i}+\alpha_{j}=N$. Then $\alpha_{i}+\alpha_{j} \geqslant N$, since $\Lambda^{1}=\cdots \Lambda^{N}=0$. Thus $x \in \mathrm{h}^{(N)}(\bar{X} \times \bar{X})$.

Let us prove the equality $\operatorname{im} p^{\mathrm{CH}}=\operatorname{im} \Phi^{N} \circ p r_{N} \circ p^{\mathrm{h}}$. One inclusion is established in Lemma 4.6. It remains to check that $\operatorname{im} \Phi^{N} \circ p r_{N} \circ p^{\mathrm{h}} \subseteq \operatorname{im} p^{\mathrm{CH}}$. By the degree formula [11, Thm 4.4.7] $\mathrm{h}(X \times X)$ is generated as a $\Lambda$-module by pushforwards $i_{\mathrm{h}}(1)$, where $i: Z \rightarrow X \times X$ is projective, $Z \in \mathbf{S m}_{k}$, and $i: Z \rightarrow i(Z)$ is birational. Following [11] we will denote such classes by $[Z \rightarrow X \times X]_{\mathrm{h}}$. Then $\mathrm{h}_{N}(X \times X)$ is additively generated by elements $\lambda[Z \rightarrow X \times X]_{\mathrm{h}}$, where $\lambda$ is homogeneous such that $\operatorname{deg} \lambda+\operatorname{codim} Z=N$. Since $\Lambda^{1}=\cdots \Lambda^{N}=0$, we have $\operatorname{codim} Z \geqslant N$.

Note that if $\operatorname{codim} Z>N$ then by Lemma $4.7 p^{\mathrm{h}}[Z \rightarrow X \times X]_{\mathrm{h}}=\left[Z_{L} \rightarrow \bar{X} \times\right.$ $\bar{X}]_{\mathrm{h}} \in \mathrm{h}^{(N+1)}(\bar{X} \times \bar{X})$. So $\operatorname{im} p r_{N} \circ p^{\mathrm{h}}$ is generated over $\Lambda^{0}$ by classes of $\left[Z_{L} \rightarrow \bar{X} \times\right.$ $\bar{X}]_{\mathrm{h}}$, where $Z \rightarrow X \times X$ has codimension $N$.

By Lemma 4.7 for any $Z \rightarrow X \times X$ of codimension $N$ we have $\Phi^{N} \circ p r_{N} \circ$ $p^{\mathrm{h}}\left([Z \rightarrow X \times X]_{\mathrm{h}}\right)=p^{\mathrm{CH}}\left([Z \rightarrow X \times X]_{\mathrm{CH}}\right)$. Then $\operatorname{im} \Phi^{N} \circ p r_{N} \circ p^{\mathrm{h}} \subseteq p^{\mathrm{CH}}$ and the lemma is proven.

Theorem B. Let $\mathrm{h}$ be an oriented cohomology theory with coefficient ring $\Lambda$. Assume that the Chow motive $\mathcal{R}$ is indecomposable over $\Lambda^{0}$ and $\Lambda^{1}=\cdots=\Lambda^{N}=0$. Then the $\mathrm{h}$-motive $\mathcal{R}_{\mathrm{h}}$ from theorem $\mathrm{A}$ is indecomposable. 
MOTIVES AND ORIENTED COHOMOLOGY OF GENERICALLY CELLULAR VARIETIES 287

Proof. By definition, $\mathcal{R}_{\mathrm{h}}=\left(X, r_{0}\right)$, where $r_{0}$ is an idempotent in $\mathrm{h}_{N}^{(N)}(X \times X)$. If $\mathcal{R}_{\mathrm{h}}$ is decomposable, then $r_{0}=r_{1}+r_{2}$ for some nontrivial orthogonal idempotents $r_{1}, r_{2} \in \mathrm{h}_{N}(X \times X)$. Then by Lemma $4.10 r_{1}, r_{2} \in \mathrm{h}_{N}^{(N)}(X \times X)$ and $p_{1}=\Phi^{N} \circ p r_{N} \circ$ $p^{\mathrm{h}}\left(r_{1}\right)$ and $p_{2}=\Phi^{N} \circ p r_{N} \circ p^{\mathrm{h}}\left(r_{2}\right)$ are rational idempotents and $p^{\mathrm{CH}}\left(p_{0}\right)=p_{1}+p_{2}$. These idempotents are orthogonal and nontrivial, since $\operatorname{ker} \Phi^{N} \circ p r_{N} \circ p^{\mathrm{h}}$ is nilpotent. Hence, the Chow motive $\mathcal{R}=\left(X, p_{0}\right)$ is decomposable, a contradiction.

Example 4.11. If $\mathrm{h}$ is $\Omega$ or connective $K$-theory, all the elements in the coefficient ring have negative degree. Then Theorems A and B prove that the $\mathrm{h}$-motivic irreducible decomposition coincides with the integral Chow-motivic decomposition. This gives another proof of the result by Vishik-Yagita [18, Cor. 2.8].

Example 4.12. Take $\mathrm{h}$ to be Morava K-theory $\mathrm{h}=K(n)^{*}$. Its coefficient ring is $\mathbb{F}_{p}\left[v_{n}, v_{n}^{-1}\right]$, where $\operatorname{deg}\left(v_{n}\right)=-2\left(p^{n}-1\right)$. In the case $n>\log _{p}\left(\frac{N}{2}+1\right)$ Theorems A and B prove that $M^{K(n)}(X)$ has the same irreducible decomposition as the Chow motive modulo $p$.

Theorem C. Suppose that $X, Y$ are generically cellular and $Y$ is a twisted form of $X$, i.e., $\bar{Y} \cong \bar{X}$.

If $M^{\mathrm{CH}}\left(X, \Lambda^{0}\right) \cong M^{\mathrm{CH}}\left(Y, \Lambda^{0}\right)$, then $M^{\mathrm{h}}(X) \cong M^{\mathrm{h}}(Y)$.

Proof. Let $f \in \mathrm{CH}^{N}(X \times Y)$ and $g \in \mathrm{CH}^{N}(Y \times X)$ be correspondences that give mutually inverse isomorphisms between $M^{\mathrm{CH}}(X)$ and $M^{\mathrm{CH}}(Y)$. Consider the diagram

$\mathrm{CH}^{N}\left(X \times Y, \Lambda^{0}\right) \stackrel{p^{\mathrm{CH}}}{\longrightarrow} \mathrm{CH}^{N}\left(\bar{X} \times \bar{X}, \Lambda^{0}\right) \stackrel{\Phi^{N}}{\longleftarrow} \mathrm{h}_{N}^{(N / N+1)}(\bar{X} \times \bar{X}) \stackrel{p r_{N} \circ p^{\mathrm{h}}}{\longleftarrow} \mathrm{h}_{N}^{(N)}(X \times Y)$.

Then by Lemma 4.6 we can find $f_{1} \in \mathrm{h}_{N}^{(N)}(X \times Y)$ and $g_{1} \in \mathrm{h}_{N}^{(N)}(Y \times X)$ such that $\Phi^{N} \circ p r_{N} \circ p^{\mathrm{h}}\left(f_{1}\right)=f$ and $\Phi^{N} \circ p r_{N} \circ p^{\mathrm{h}}\left(g_{1}\right)=g$. Then $g_{1} \circ f_{1}-i d_{X}$ lies in the kernel of the map

$$
\mathrm{h}_{N}^{(N)}(X \times X) \stackrel{p r_{N} \circ p^{\mathrm{h}}}{\longrightarrow} \mathrm{h}_{N}^{(N / N+1)}(\bar{X} \times \bar{X}),
$$

which consists of nilpotents by Lemma 4.8. So $g_{1} \circ f_{1}-i d_{X}$ is nilpotent. By the same reasons $f_{1} \circ g_{1}-i d_{Y}$ is nilpotent. Then $M^{\mathrm{h}}(X)$ and $M^{\mathrm{h}}(Y)$ are isomorphic by Lemma 4.9.

\section{References}

[1] F. Anderson and K. Fuller, Rings and categories of modules, Springer-Verlag, New York, 1992.

[2] A. Borel, Sur la cohomologie des espaces homogénes des groupes de Lie compacts, C.R. Acad. Sci 233 (1951), 569-571.

[3] N. Bourbaki, Elements of mathematics. Commutative algebra, Addison-Wesley Publishing Co., Reading, Mass., 1972.

[4] B. Calmés, V. Petrov, and K. Zainoulline, Invariants, torsion indices and oriented cohomology of complete flags, Ann. Sci. Éc. Norm. Supér 46, no. 3 (2013), 405-448.

[5] R. Elman, N. Karpenko, and A. Merkurjev, The algebraic and geometric theory of quadratic forms, American Mathematical Society, Providence, RI, 2008. 
[6] S. Garibaldi and K. Zainoulline, The $\gamma$-filtration and the Rost invariant, J. Reine Angew. Math, to appear (2013).

[7] S. Gille and K. Zainoulline, Equivariant pretheories and invariants of torsors, Transform. Groups 17, no. 2 (2012), 471-498.

[8] M. Hazewinkel, Formal groups and applications, Academic Press, Inc., New York-London, 1978.

[9] J. Hornbostel and V. Kiritchenko, Schur calculus for algebraic cobordism, J. Reine Angew. Math. 656 (2011), 59-85.

[10] V. Kac, Torsion in cohomology of compact Lie groups and Chow rings of reductive algebraic groups, Invent. Math 80, no. 1 (1985), 74-119.

[11] M. Levine and F. Morel, Algebraic cobordism, Springer, Berlin, 2007.

[12] Ju. Manin, Correspondences, motifs and monoidal transformations, Math. Sb. (N.S.) 77 (1968), 475-507.

[13] R. Marlin, Anneaux de Chow des groupes algebriques $S O(n), \operatorname{Spin}(n), G_{2}$ et $F_{4}$, Publications Mathématiques d'Orsay 95 (1974), 74-119.

[14] A. Nenashev and K. Zainoulline, Oriented cohomology and motivic decompositions of relative cellular spaces, J. Pure Appl. Algebra 205, no. 2 (2006), 323-340.

[15] I. Panin, Oriented cohomology theories of algebraic varieties II (After I. Panin and A. Smirnov), Homology, Homotopy, Appl 11, no. 1 (2009), 349-405.

[16] I. Panin, Splitting principle and K-theory of simply connected semisimple algebraic groups, St. Petersburg Math. J. 10, no. 1 (1999), 69-101.

[17] V. Petrov, N. Semenov, and K. Zainoulline, J-invariant of linear algebraic groups, Ann. Sci. Éc. Norm. Supér (4)41, no. 6 (2008), 1023-1053.

[18] A. Vishik and N. Yagita, Algebraic cobordism of a Pfister quadric, J. Lond. Math. Soc (2)76, no. 3 (2007), 586-604.

[19] A. Vishik and K. Zainoulline, Motivic splitting lemma, Doc. Math. 13 (2008), $81-96$.

[20] N. Yagita, Algebraic cobordism of simply connected Lie groups, Math. Proc. Cambridge Philos. Soc. 139, no. 2 (2005), 243-260.

[21] K. Zainoulline, Twisted gamma filtration of a linear algebraic group, Compos. Math. 148, no. 5 (2012), 1645-1654.

Alexander Neshitov anesh094@uottawa.ca

Department of Mathematics and Statistics, University of Ottawa, 585 King Edward, Ottawa, ON K1N 6N5, Canada

St. Petersburg Department of Steklov Mathematical Institute 27 Fontanka, St.Petersburg, 191023, Russia 